H. UEDA

KODAI MATH. J.

5 (1982), 289-302

\title{
AN ENTIRE FUNCTION RELATED TO THEOREMS OF BARRY
}

\author{
BY HIDEHARU UEDA
}

\section{Introduction.}

Let $f(z)$ be an entire function of order $\rho$ and lower order $\mu$, where $0 \leqq \mu \leqq$ $\rho \leqq 1$. The classical $\cos \pi \rho$ theorem of Wiman and Valiron states that, given $\varepsilon>0$, the inequality

$$
\log m^{*}(r, f)>(\cos \pi \rho-\varepsilon) \log M(r, f)
$$

holds for a sequence $r=r_{n} \rightarrow \infty$, where

$$
m^{*}(r, f)=\min _{|z|=r}|f(z)|, \quad M(r, f)=\max _{|z|=r}|f(z)| .
$$

This was sharpened by Kjellberg [5], who showed that (1) holds with $\rho$ replaced by $\mu(<1)$, independently of the value of $\rho$.

Much work including the above has been performed related to the $\cos \pi \rho$ theorem. The starting point of the considerations presented here is the following results due to Barry.

Theorem A. ([1]) If $\rho<\alpha<1$, and if

$$
E=\left\{r ; \log m^{*}(r, f)>\cos \pi \alpha \log M(r, f)\right\},
$$

then

$$
\underline{\log \text { dens }} E \geqq 1-\rho / \alpha \text {. }
$$

THEOREM B. ([2]) If $\mu<\alpha<1$, and if $E$ is defined by (2), then

$$
\overline{\log \operatorname{dens}} E \geqq 1-\mu \backslash \alpha \text {. }
$$

The estimates (3) and (4) are both sharp in the sense that the sign $\geqq$ cannot be replaced by $>$. In fact, the following theorem was proved by Hayman.

TheOREM C. ([4, Theorem 1.]) Given any numbers $\rho, \alpha$, such that $0<\rho<\alpha<1$, there exists an entire function $f(z)$ of order $\rho$ and regular growth such that

$$
\underline{\log \operatorname{dens}} E=\overline{\log \operatorname{dens}} E=1-\rho / \alpha,
$$

Received February 9, 1981 
where $E$ is the set defined by (2).

The function $f(z)$ in Theorem $C$ satisfies both (3) and (4) with the sign of equality. Motivated by this fact, the following problem is naturally raised.

Problem. Let $\mu, \rho, \alpha$ be any numbers such that $0 \leqq \mu \leqq \rho<\alpha<1$. Then is it possible to construct an entire function $f(z)$ of order $\rho$ and lower order $\mu$ such that

$$
1-\rho / \alpha=\underline{\log \operatorname{dens}} E \leqq \overline{\log \operatorname{dens}} E=1-\mu / \alpha,
$$

where $E$ is the set defined by (2)?

Observe first that for entire functions $f(z)$ of order 0 , the Barry's estimates (3) and (4) imply $\log \operatorname{dens} E=1$, so that our problem is solved affirmatively for $\mu=\rho=0$. And since, for $0<\mu=\rho<\alpha<1$, Hayman has given examples satisfying the conclusion of our problem, we may consider the case $0 \leqq \mu<\rho<\alpha<1$.

In this paper we prove the following

THEOREM. Given any numbers $\mu, \rho$, such that $0 \leqq \mu<\rho<\alpha<1$, there exists an entire function $f(z)$ of order $\rho$ and lower order $\mu$ and such that

$$
1-\rho / \alpha=\underline{\log \operatorname{dens}} E<\overline{\log \operatorname{dens}} E=1-\mu / \alpha,
$$

where $E$ is defined by (2).

All the above results combine to show that our problem is solved affirmatively in all cases.

In $\S \S 1-4$, we suppose $\mu>0$; a special argument when $\mu=0$ is in $\S 5$.

\section{Construction of a continuous increasing function $\nu(t)$.}

Let $l$ be the positive number satisfying

$$
\rho=\frac{\mu \alpha(l+1)}{\alpha+\mu l} \text {. }
$$

Define a sequence $\left\{r_{m}\right\}_{0}^{\infty}$ by

$$
r_{0}=1, \quad r_{m}=3^{(l+1) m-1} \quad(m \geqq 1) .
$$

Further let $\left\{\alpha_{m}\right\}_{0}^{\infty}$ be a decreasing sequence tending to $\alpha$ such that $\alpha_{0}<1$, and let $\left\{r_{m}^{\prime}\right\}_{0}^{\infty}$ be an increasing sequence defined by

$$
\left(\frac{r_{m}^{\prime}}{r_{m}}\right)^{\alpha}=\left(\frac{r_{m+1}}{r_{m}}\right)^{\mu}
$$

Then, since $\mu<\alpha \leqq \alpha_{m}$, we deduce from (1.2) and (1.3) that

$$
r_{m}<r_{m}^{\prime}<r_{m+1} \quad(m=0,1,2, \cdots) .
$$

Now, we define a nonnegative function $\lambda(t)(t \geqq 1)$ as follows: 


$$
\lambda(t)= \begin{cases}\alpha_{m} & \left(r_{m} \leqq t \leqq r_{m}^{\prime} ; m=0,1,2, \cdots\right) \\ 0 & \left(r_{m}^{\prime}<t<r_{m+1} ; m=0,1,2, \cdots\right) .\end{cases}
$$

Then corresponding to $\lambda(t)$, we take a continuous increasing function $\nu(r)(r \geqq 1)$ with

$$
\nu(r)=\exp \left(\int_{1}^{r} \lambda(t) t^{-1} d t\right)
$$

Here we show the following

LEMMA 1. The order and lower order of $\nu(r)$ are equal to $\rho$ and $\mu$, respectrvely.

Proof. Consider the interval $r_{m} \leqq r<r_{m+1}(m=0,1,2, \cdots)$. By (1.5)

$$
\log \nu(r)=\int_{1}^{r} \lambda(t) t^{-1} d t
$$

Hence, if $r_{m} \leqq r \leqq r_{m}^{\prime}$, we deduce from (1.4), (1.3) and (1.2) that

$$
\begin{aligned}
\log \nu(r) & =\sum_{s=0}^{m-1} \int_{r_{s}}^{r_{s}^{\prime}} \frac{\alpha_{s}}{t}-d t+\int_{r_{m}}^{r} \frac{\alpha_{m}}{t}-d t \\
& =\sum_{s=0}^{m-1} \alpha_{s} \log \left(\frac{r_{s}^{\prime}}{r_{s}}\right)+\alpha_{m} \log \left(\frac{r}{r_{m}}\right) \\
& =\sum_{s=0}^{m-1} \mu \log \left(\frac{r_{s+1}}{r_{s}}\right)+\alpha_{m} \log \left(\frac{r}{r_{m}}\right) \\
& =\mu \log r_{m}+\alpha_{m} \log \left(\frac{r}{r_{m}}\right) .
\end{aligned}
$$

Similarly, if $r_{m}^{\prime} \leqq r<r_{m+1}$, we deduce that

Thus

$$
\begin{aligned}
\log \nu(r) & =\sum_{s=0}^{m} \int_{r_{s}}^{r_{s}^{\prime}} \frac{\alpha_{s}}{t} d t \\
& =\mu \log r_{m+1} .
\end{aligned}
$$

$$
\frac{\log \nu(r)}{\log r}= \begin{cases}\alpha_{m}-\left(\alpha_{m}-\mu\right) \frac{\log r_{m}}{\log r} & \left(r_{m} \leqq r \leqq r_{m}^{\prime} ; m=0,1,2, \cdots\right) \\ \mu \frac{\log r_{m+1}}{\log r} & \left(r_{m}^{\prime} \leqq r<r_{m+1} ; m=0,1,2, \cdots\right) .\end{cases}
$$

From this, we see that

$$
\varliminf_{r \rightarrow \infty} \frac{\log \nu(r)}{\log r}=\varliminf_{m \rightarrow \infty} \frac{\log \nu\left(r_{m}\right)}{\log r_{m}}=\mu,
$$

and 


$$
\varlimsup_{r \rightarrow \infty} \frac{\log \nu(r)}{\log r}=\varlimsup_{m \rightarrow \infty} \frac{\log \nu\left(r_{m}^{\prime}\right)}{\log r_{m}^{\prime}}=\varlimsup_{m \rightarrow \infty} \mu \frac{\log r_{m+1}}{\log r_{m}^{\prime}} .
$$

It remains to compute $\log r_{m+1} / \log r_{m}^{\prime}$. We have

$$
\frac{\log r_{m+1}}{\log r_{m}^{\prime}}=\frac{\log r_{m+1}}{\log r_{m}+\log \left(r_{m}^{\prime} / r_{m}\right)} \text {. }
$$

Using (1.2) and (1.3), we obtain

$$
\begin{aligned}
\frac{\log r_{m+1}}{\log r_{m}^{\prime}} & =\frac{(l+1)^{m} \log 3}{(l+1)^{m-1} \log 3+\frac{\mu}{\alpha_{m}} l(l+1)^{m-1} \log 3} \\
& =\frac{l+1}{1+\frac{\mu}{\alpha_{m}} l} \\
& =\frac{\alpha_{m}(l+1)}{\alpha_{m}+\mu l} .
\end{aligned}
$$

Since $\alpha_{m} \rightarrow \alpha$ as $m \rightarrow \infty$, it follows from (1.8), (1.7) and (1.1) that

$$
\varlimsup_{r \rightarrow \infty} \frac{\log \nu(r)}{\log r}=\frac{\mu \alpha(l+1)}{\alpha+\mu l}=\rho .
$$

This proves Lemma 1.

\section{A set $F$ on the positive real axis.}

Set

$$
K_{m}^{\prime}=\frac{r_{m+1}}{r_{m}}=3^{l(l+1) m-1} \quad(m \geqq 1),
$$

and define

$$
K_{m}=\left(\log K_{m}^{\prime}\right)^{2 / \mu} .
$$

In view of (1.3), (2.1) and (2.2), we have $r_{m}^{\prime} / K_{m}>K_{m} r_{m}\left(m \geqq m_{0}\right)$

Now let

$$
F=\bigcup_{m=m_{0}}^{\infty}\left[K_{m} r_{m}, r_{m}^{\prime} / K_{m}\right] .
$$

Then we have the following

LEMmA 2. $\overline{\log \operatorname{dens}} F \geqq \rho / \alpha, \underline{\log \operatorname{dens}} F \geqq \mu / \alpha$.

Proof. Let $R$ be a large positive number and let $m_{1}$ be the integer such that $r_{m_{1}}^{\prime} / K_{m_{1}} \leqq R<r_{m_{1}+1}^{\prime} / K_{m_{1}+1}$. Suppose first that $r_{m_{1}}^{\prime} / K_{m_{1}} \leqq R<K_{m_{1}+1} r_{m_{1}+1}$ and $m_{1} \geqq m_{0}$. Then we have from (2.3), (1.3) and (2.1) that 


$$
\begin{aligned}
\int_{F \cap[1, R]} \frac{d t}{t} & =\sum_{m=m_{0}}^{m_{1}} \int_{K_{m} r_{m}}^{r_{m}^{\prime} / K_{m}} \frac{d t}{t}=\sum_{m=m_{0}}^{m_{1}}\left\{\log \left(\frac{r_{m}^{\prime}}{r_{m}}\right)-2 \log K_{m}\right\} \\
& =\sum_{m=m_{0}}^{m_{1}}\left\{\frac{\mu}{\alpha_{m}} \log K_{m}^{\prime}-2 \log K_{m}\right\} .
\end{aligned}
$$

In view of (2.2)

$$
\log K_{m}=o\left(\log K_{m}^{\prime}\right) \quad(m \rightarrow \infty) .
$$

Also $\alpha_{m} \rightarrow \alpha$ as $m \rightarrow \infty$. Hence given $\varepsilon>0$, we can choose $N=N(\varepsilon)$, so that for $m_{1} \geqq N$

$$
\int_{F \cap[1, R]} \frac{d t}{t}>\frac{\mu}{\alpha}(1-\varepsilon) \sum_{m=N}^{m_{1}} \log K_{m}^{\prime}=\frac{\mu}{\alpha}(1-\varepsilon) \log \frac{r_{m_{1}+1}}{r_{N}} .
$$

Since $R<K_{m_{1}+1} r_{m_{1}+1}$, it follows from (1.2), (2.1) and (2.4) that

$$
\begin{aligned}
\frac{\log r_{m_{1}+1}}{\log R} & >\frac{\log r_{m_{1}+1}}{\log \left(K_{m_{1}+1} r_{m_{1}+1}\right)}=\frac{\log r_{m_{1}+1}}{\log K_{m_{1}+1}+\log r_{m_{1}+1}} \\
& =\frac{\log r_{m_{1}+1}}{(1+o(1)) \log r_{m_{1}+1}} \quad\left(m_{1} \rightarrow \infty\right) \\
& >1-\varepsilon \quad\left(m_{1} \geqq N_{1}(\varepsilon)\right) .
\end{aligned}
$$

Thus for all sufficiently large $R \in \bigcup_{m=0}^{\infty}\left[r_{m}^{\prime} / K_{m}, K_{m+1} r_{m+1}\right]$

$$
\frac{1}{\log R} \int_{F \cap[1, R]} \frac{d t}{t}>\frac{\mu}{\alpha}(1-\varepsilon)^{3} .
$$

Next suppose that $K_{m_{1}+1} r_{m_{1}+1} \leqq R<r_{m_{1}+1}^{\prime} / K_{m_{1}+1}$ and $m_{1} \geqq m_{0}$. In this case, we have from (2.3), (1.3), (2.1) and (2.5) that

$$
\begin{aligned}
\int_{F \cap[1, R]} \frac{d t}{t} & =\sum_{m=m_{0}}^{m_{1}}\left\{\frac{\mu}{\alpha_{m}} \log K_{m}^{\prime}-2 \log K_{m}\right\}+\log \frac{R}{K_{m_{1}+1} r_{m_{1}+1}} \\
& >\frac{\mu}{\alpha}(1-\varepsilon) \log \frac{r_{m_{1}+1}}{r_{N}}+\log \frac{R}{K_{m_{1}+1} r_{m_{1}+1}} \quad\left(m_{1} \geqq N\right) .
\end{aligned}
$$

Since $K_{m_{1}+1} r_{m_{1}+1} \leqq R$, it follows from (1.2), (2.1) and (2.4) that

$$
\begin{aligned}
& \frac{\log R-\log K_{m_{1}+1}-(1-(\mu / \alpha)(1-\varepsilon)) \log r_{m_{1}+1}-(\mu / \alpha)(1-\varepsilon) \log r_{N}}{\log R} \\
& >1-o(1)-(1-(\mu / \alpha)(1-\varepsilon)) \frac{\log r_{m_{1}+1}}{\log R} \quad\left(m_{1} \rightarrow \infty\right) \\
& \geqq 1-o(1)-(1-(\mu / \alpha)(1-\varepsilon)) \frac{\log r_{m_{1}+1}}{\log K_{m_{1+1} r_{m_{1}+1}}} \\
& >1-\varepsilon-(1-(\mu / \alpha)(1-\varepsilon))(1-\varepsilon) \quad\left(m_{1} \geqq N_{2}(\varepsilon)\right)
\end{aligned}
$$




$$
=(\mu / \alpha)(1-\varepsilon)^{2} .
$$

Thus for all sufficiently large $R \in \bigcup_{m=0}^{\infty}\left[K_{m+1} r_{m+1}, r_{m+1}^{\prime} / K_{m+1}\right]$

$$
\frac{1}{\log R} \int_{F \cap[1, R]} \frac{d t}{t}>\frac{\mu}{\alpha}(1-\varepsilon)^{2} .
$$

Together, (2.6) and (2.7) give

$$
\frac{1}{\log R} \int_{F \cap[1, R]} \frac{d t}{t}>\frac{\mu}{\alpha}(1-\varepsilon)^{3}
$$

for all sufficiently large $R$, i.e.

$$
\underline{\log \operatorname{dens}} F \geqq \frac{\mu}{\alpha}(1-\varepsilon)^{3} .
$$

Since $\varepsilon$ is an arbitrary positive number independent of $F$, we have

$$
\underline{\log \operatorname{dens}} F \geqq-\frac{\mu}{\alpha} .
$$

In order to show that $\overline{\log \operatorname{dens}} F \geqq \rho / \alpha$, we put $R=r_{m_{1}}^{\prime} / K_{m_{1}} \equiv R_{m_{1}}$ in (2.5). Then from (2.1), (1.3), (2.4) and (1.1) it follows that

$$
\begin{aligned}
& \frac{1}{\log R_{m_{1}}} \int_{F \cap\left[1, R_{\left.m_{1}\right]}\right]} \frac{d t}{t}>\frac{\mu}{\alpha}(1-\varepsilon) \frac{\log r_{m_{1}+1}-\log r_{N}}{\log r_{m_{1}}^{\prime}-\log K_{m_{1}}} \\
& =\frac{\mu}{\alpha}(1-\varepsilon) \frac{\log r_{m_{1}}+\log K_{m_{1}}^{\prime}-\log r_{N}}{\log r_{m_{1}}+\frac{\mu}{\alpha_{m_{1}}} \log K_{m_{1}}^{\prime}-\log K_{m_{1}}} \\
& =\frac{\mu}{\alpha}(1-\varepsilon) \frac{(l+1)^{m_{1}-1} \log 3+l(l+1)^{m_{1}-1} \log 3-O(1)}{(l+1)^{m_{1}-1} \log 3+\left(\frac{\mu}{\alpha}-o(1)\right) l(l+1)^{m_{1}-1} \log 3} \quad\left(m_{1} \rightarrow \infty\right) \\
& >\frac{\mu}{\alpha}(1-\varepsilon)^{2} \frac{1+l}{1+\frac{\mu}{\alpha} l} \quad\left(m_{1}>N_{3}(\varepsilon)\right) \\
& =\frac{\mu(1+l)}{\alpha+\mu l}(1-\varepsilon)^{2}=\frac{\rho}{\alpha}(1-\varepsilon)^{2} .
\end{aligned}
$$

Thus

$$
\overline{\log \operatorname{dens}} F \geqq \varlimsup_{m_{1} \rightarrow \infty} \frac{1}{\log R_{m_{1}}} \int_{F \cap\left[1, R_{m_{1}}\right]} \frac{\alpha t}{t} \geqq \frac{\rho}{\alpha}(1-\varepsilon)^{2} .
$$

Agoin, since $\varepsilon$ is an arbitrary positive number independent of $F$, we obtain

$$
\overline{\log \operatorname{dens}} F \geqq-\frac{\rho}{\alpha} .
$$

This completes the proof of Lemma 2. 


\section{An entire function $f(z)$ of genus zero associated with $[\nu(t)]$.}

Let $f(z)$ be a canonical product all of whose zeros $\left\{a_{n}\right\}_{1}^{\infty}$ are real and negative and such that

$$
n(t) \equiv n(t, 0)= \begin{cases}0 & (t<1) \\ {[\nu(t)]} & (t \geqq 1) .\end{cases}
$$

It follows from Lemma 1.4 in [3], Lemma 1 and (3.1) that

$$
\sum_{k=1}^{\infty} \frac{1}{\left|a_{k}\right|}=\int_{1}^{\infty} \frac{n(t)}{t^{2}} d t<\int_{1}^{\infty} \frac{\nu(t)}{t^{2}} d t<\infty .
$$

This implies that $f(z)$ has genus zero, and so for $|\arg z|<\pi$ we have $[3, \mathrm{p} 21]$

$$
\log f(z)=z \int_{1}^{\infty} \frac{n(t)}{t(t+z)} d t
$$

First we prove the following

LEMMA 3. $f(z)$ has order $\rho$ and lower order $\mu$.

Proof. We denote the order and lower order of $f(z)$ by $\rho_{f}$ and $\mu_{f}$, respectively. Take $\varepsilon>0$ small so that $0<\mu-\varepsilon<\rho+\varepsilon<1$. By Lemma 1

$$
t^{\mu-\varepsilon} \leqq n(t) \leqq t^{\rho+\varepsilon} \quad\left(t \geqq t_{0} \equiv t_{0}(\varepsilon) \geqq 1\right) .
$$

From (3.2) and (3.3) it follows that

Similarly

$$
\begin{aligned}
\log M(r, f) & =r \int_{1}^{\infty} \frac{n(t)}{t(t+r)} d t \\
& \leqq r \int_{1}^{t_{0}} \frac{n\left(t_{0}\right)}{t(t+r)} d t+r \int_{0}^{\infty} \frac{t^{\rho+\varepsilon}}{t(t+r)} d t \\
& =n\left(t_{0}\right) \log \frac{t_{0}(1+r)}{t_{0}+r}+r^{\rho+\varepsilon} \int_{0}^{\infty} \frac{u^{\rho+\varepsilon-1}}{u+1} d u \\
& =O\left(r^{\rho+\varepsilon}\right) \quad(r \rightarrow \infty) .
\end{aligned}
$$

$$
\begin{aligned}
\log M(r, f) & \geqq r \int_{t_{0}}^{\infty} \frac{t^{\mu-\varepsilon}}{t(t+r)} d t \\
& =r^{\mu-\varepsilon} \int_{t_{0} / r}^{\infty} \frac{u^{\mu-\varepsilon-1}}{u+1} d u \\
& \geqq r^{\mu-\varepsilon} \int_{1}^{\infty} \frac{u^{\mu-\varepsilon-1}}{2 u} d u \quad\left(r \geqq t_{0}\right) \\
& =\frac{1}{2} \frac{r^{\mu-\varepsilon}}{1+\varepsilon-\mu}=O\left(r^{\mu-\varepsilon}\right) \quad(r \rightarrow \infty) .
\end{aligned}
$$


Since we can choose $\varepsilon(>0)$ arbitrarily small, we deduce that

$$
\mu \leqq \mu_{f} \leqq \rho_{f} \leqq \rho .
$$

Next, we proceed to show that $\rho_{f} \geqq \rho$. For this purpose, note that $N(t, 0)$ has the same order as $n(t)$, and so by Lemma 1 it has order $\rho$. Further the first fundamental theorem gives $T(t, f) \geqq N(t, 0)$. Thus we have $\rho_{f} \geqq \rho$.

It remains to prove that $\mu_{f} \leqq \mu$. The proof is a little more complicated. Set

$$
R=r_{m} / K_{m-1} \equiv R_{m},
$$

and write $\log M(R, f)$ as follows:

$$
\begin{aligned}
\log M(R, f) & =R\left(\int_{1}^{r_{m-1}^{\prime}}+\int_{r_{m-1}^{\prime}}^{r_{m}}+\int_{r_{m}}^{\infty}\right) \frac{n(t)}{t(t+R)} d t \\
& \equiv I_{1}+I_{2}+I_{3} .
\end{aligned}
$$

Using (1.3) and (2.4), we have $R_{m} \geqq r_{m-1}^{\prime}\left(m \geqq m_{2}\right)$. It is clear that

$$
I_{3} \leqq R \int_{r_{m}}^{\infty} \frac{\nu(t)}{t^{2}} d t
$$

It is a consequence of (1.6) that $\nu(t) / t^{\alpha_{0}}$ decreases for all $t \geqq 1$. Thus

$$
\begin{aligned}
I_{3} & \leqq R \int_{r_{m}}^{\infty} \frac{\nu(R)}{R^{\alpha_{0}}} t^{\alpha_{0}} \frac{d t}{t^{2}}=R^{1-\alpha_{0}} \nu(R) \frac{1}{1-\alpha_{0}}\left(\frac{1}{r_{m}}\right)^{1-\alpha_{0}} \\
& =\frac{1}{1-\alpha_{0}}\left(\frac{1}{K_{m-1}}\right)^{1-\alpha_{0}} r_{m}^{\mu} \quad\left(m \geqq m_{2}\right) .
\end{aligned}
$$

Now, by (1.6) and (3.1), $n(t)=\left[r_{m}^{\mu}\right]$ for $r_{m-1}^{\prime} \leqq t \leqq r_{m}$. Thus, from (2.1), (1.3) and (2.4) it follows that

$$
\begin{aligned}
I_{2} & =\left[r_{m}^{\mu}\right] \log \frac{r_{m}}{r_{m-1}^{\prime}} \frac{r_{m-1}^{\prime}+R}{r_{m}+R} \\
& =\left[r_{m}^{\mu}\right] \log \frac{1+\frac{1}{K_{m-1}} \frac{r_{m}}{r_{m-1}^{\prime}}}{1+\frac{1}{K_{m-1}}} \\
& =\left[r_{m}^{\mu}\right] \log \frac{1+\frac{1}{K_{m-1}}\left(K_{m-1}^{\prime}\right)^{1-(\mu / \alpha m-1)}}{1+\frac{1}{K_{m-1}}} \\
& \sim\left(1-\frac{\mu}{\alpha}\right)\left(\log K_{m-1}^{\prime}\right) r_{m}^{\mu} \quad(m \rightarrow \infty) .
\end{aligned}
$$

Finally, by (1.6), (2.1) and (1.3) 


$$
\begin{aligned}
I_{1} & \leqq \int_{1}^{r_{m-1}^{\prime}} \frac{\nu(t)}{t} d t \leqq \sum_{s=1}^{m-1} \nu\left(r_{s}\right) \log \left(\frac{r_{s}}{r_{s-1}}\right)+\int_{r_{m-1}}^{r_{m-1}^{\prime}} \frac{r_{m-1}^{\mu}\left(t / r_{m-1}\right)^{\alpha} m-1}{t} d t \\
& <\frac{r_{m-1}^{\mu}}{\alpha_{m-1}}\left\{\left(\frac{r_{m-1}^{\prime}}{r_{m-1}}\right)^{\alpha-1}-1\right\}+\left(\log K_{m-2}^{\prime}\right) \sum_{s=1}^{m-1} r_{s}^{\mu} \\
& <\frac{r_{m}^{\mu}}{\alpha_{m-1}}+\left(\log K_{m-2}^{\prime}\right) r_{m}^{\mu} \sum_{s=1}^{m-1}\left(\frac{r_{s}}{r_{m}}\right)^{\mu} .
\end{aligned}
$$

Also in view of (2.4)

$$
\frac{r_{j}}{r_{j+1}}=\frac{1}{K_{j}^{\prime}}=\frac{1}{3^{l(l+1)^{j-1}}}<\frac{1}{3} \quad\left(j>\jmath_{0}(l)\right) .
$$

Further, it is easy to see that

$$
\sum_{s=1}^{\infty}\left(\frac{1}{3}\right)^{\mu s}=\frac{1}{3^{\mu}-1}<\frac{1}{\mu} .
$$

Thus

$$
\begin{aligned}
I_{1} & <\frac{r_{m}^{\mu}}{\alpha_{m-1}}+\left(\log K_{m-2}^{\prime}\right)\left\{\sum_{s>\jmath_{0}}^{m-1}\left(\frac{1}{3}\right)^{\mu(m-s)}+\jmath_{0}\right\} r_{m}^{\mu} \\
& <\frac{r_{m}^{\mu}}{\alpha_{m-1}}+\left(\frac{1}{\mu}+\jmath_{0}\right)\left(\log K_{m-2}^{\prime}\right) r_{m}^{\mu} .
\end{aligned}
$$

Substituting (3.5)-(3.7) into (3.4), we obtain

$$
\begin{aligned}
\log M(R, f) & <\left\{o(1)+O\left(\log K_{m-1}^{\prime}\right)+O\left(\log K_{m-2}^{\prime}\right)\right\} r_{m}^{\mu} \\
& =r_{m}^{\mu} O\left(\log K_{m-1}^{\prime}\right) \\
& =r_{m}^{\mu} O\left(\log r_{m-1}\right)=r_{m}^{\mu} O\left(\log r_{m}\right) \quad(m \rightarrow \infty) .
\end{aligned}
$$

Therefore by (1.2), (2.1) and (2.4)

$$
\frac{\log \log M(R, f)}{\log R}<\frac{(1+o(1)) \mu \log r_{m}}{\log r_{m}-\log K_{m-1}}=(1+o(1)) \mu \quad(m \rightarrow \infty) .
$$

This shows that $\mu_{f} \leqq \mu$. This completes the proof of Lemma 3 .

Now, we choose $\left\{\alpha_{m}\right\}_{0}^{\infty}$ as follows:

$$
\alpha_{m}=\alpha+\frac{1-\alpha}{2} \frac{1}{(l+1)^{\sqrt{ } m+1}} .
$$

Here we show the following

LEMma 4. For all sufficiently large $r \in F$

$$
\log m^{*}(r, f)<\cos \pi \alpha \log M(r, f) .
$$

Proof. We make use of Lemma 3 in [4]. Because of (1.2), (3.8), (1.3), (1.4), (1.5) and (2.2), this lemma is applicable to our $f(z)$. Hence we deduce that for 


$$
\begin{aligned}
r \in\left[K_{m} r_{m}, r_{m}^{\prime} / K_{m}\right] & \\
& \log M(r, f) \geqq \nu(r)\left\{\frac{\pi}{\sin \pi \alpha_{m}}+O\left(\frac{1}{K_{m}^{\delta}}\right)\right\}+O(\log r), \\
& \log m^{*}(r, f) \leqq \nu(r)\left\{\pi \cot \pi \alpha_{m}+O\left(\frac{1}{K_{m}^{\delta}}\right)\right\}+O(\log r),
\end{aligned}
$$

where $\delta \in(0,1]$ is a constant depending only on $\alpha_{0}$ and $\mu$.

Therefore by (2.1), (2.2), Lemma 1 and (3.8)

$$
\begin{aligned}
\frac{\log m^{*}(r, f)}{\log M(r, f)} & \leqq \cos \pi \alpha_{m}+O\left(\frac{1}{K_{m}^{\delta}}\right)+O\left(\frac{\log r}{\nu(r)}\right) \\
& =\cos \pi \alpha_{m}+O\left\{\frac{1}{(l+1)^{(m-1) 2 \delta / \mu}}\right\} \\
& =\cos \pi \alpha_{m}+o\left(\alpha_{m}-\alpha\right) \quad(m \rightarrow \infty) \\
& <\cos \pi \alpha \quad\left(m \geqq m_{3}\right) .
\end{aligned}
$$

This gives the desired result.

\section{Proof of Theorem; the case $\mu>0$.}

Define $E$ by (2). Then by Lemma $4 E \cap F \cap(R, \infty)=\phi$ for all sufficiently large $R$. Hence

$$
\overline{\log \operatorname{dens}}(E+F) \leqq 1
$$

so that

(4.1) $\quad \underline{\log \operatorname{dens}} E+\overline{\log \operatorname{dens}} F \leqq 1, \quad \overline{\log \operatorname{dens}} E+\underline{\log \operatorname{dens}} F \leqq 1$.

It follows from (4.1) and Lemma 2 that

$$
\underline{\log \operatorname{dens}} E \leqq 1-\rho / \alpha, \quad \overline{\log \operatorname{dens}} E \leqq 1-\mu / \alpha .
$$

Now, we use Lemma 3 and Theorem A or Theorem B to obtain

$$
\underline{\log \operatorname{dens}} E \geqq 1-\rho / \alpha, \quad \overline{\log \operatorname{dens}} E \geqq 1-\mu / \alpha .
$$

Combining (4.2) and (4.3), we have

$$
1-\rho / \alpha=\underline{\log \operatorname{dens}} E<\overline{\log \operatorname{dens}} E=1-\mu / \alpha .
$$

This is the desired result.

5. The case $\mu=0$.

For given $\rho$ and $\alpha$, we put 


$$
L=\alpha \rho /(\alpha-\rho),
$$

and define three sequences $\left\{r_{m}\right\}_{1}^{\infty},\left\{\alpha_{m}\right\}_{1}^{\infty},\left\{\mu_{m}\right\}_{1}^{\infty}$ by

$$
r_{m}=3^{m !}, \quad \alpha_{m}=\alpha+\frac{1-\alpha}{2} \frac{1}{m+1}, \quad \mu_{m}=L / m .
$$

Further let $\left\{r_{m}^{\prime}\right\}_{1}^{\infty}$ be a sequence defined by

$$
\left(\frac{r_{m}^{\prime}}{r_{m}}\right)^{\alpha m}=\left(\frac{r_{m+1}}{r_{m}}\right)^{\mu_{m}}
$$

Since $\alpha_{m} \rightarrow \alpha$ and $\mu_{m} \rightarrow 0$, there exists a positive integer $m_{3}$ such that $m \geqq m_{3}$ implies $\mu_{m}<\alpha_{m}$, so we deduce that $r_{m}<r_{m}^{\prime}<r_{m+1}\left(m \geqq m_{3}\right)$. Now, we define a nonnegative function $\lambda(t)\left(t \geqq r_{m_{3}}\right)$ by $(1.4)$ and set

$$
\nu(r)=\exp \left(\int_{r_{m_{3}}}^{r} \lambda(t) t^{-1} d t\right) \quad\left(r \geqq r_{m_{3}}\right) .
$$

LEMMA 5. The order and lower order of $\nu(r)$ are equal to $\rho$ and 0 , respectrvely.

Proof. Consider the interval $r_{m} \leqq r<r_{m+1}\left(m \geqq m_{3}\right)$. As in the proof of Lemma 1 , we have for $r_{m} \leqq r \leqq r_{m}^{\prime}$

$$
\begin{aligned}
\log \nu(r) & =\sum_{s=m_{3}}^{m-1} \mu_{s} \log \left(\frac{r_{s+1}}{r_{s}}\right)+\alpha_{m} \log \left(\frac{r}{r_{m}}\right) \\
& =L \log 3 \sum_{s=m_{3}}^{m-1} s^{1}+\alpha_{m} \log \left(\frac{r}{r_{m}}\right) \\
& =\alpha_{m} \log r-\left\{\alpha_{m}-L \frac{\sum_{s=m_{3}}^{m-1} s !}{m !}\right\} \log r_{m},
\end{aligned}
$$

and for $r_{m}^{\prime} \leqq r<r_{m+1}$

$$
\log \nu(r)=\sum_{s=m_{3}}^{m} \mu_{s} \log \left(\frac{r_{s+1}}{r_{s}}\right)=L \log 3 \sum_{s=m_{3}}^{m} s !=L \frac{\sum_{s=m_{3}}^{m} s !}{m !} \log r_{m} .
$$

Hence

$$
\begin{aligned}
\varliminf_{r \rightarrow \infty} \frac{\log \nu(r)}{\log r} & =\varliminf_{m \rightarrow \infty} L \frac{\sum_{s=m_{3}}^{m-1} s !}{m !}=0, \\
\varlimsup_{r \rightarrow \infty} \frac{\log \nu(r)}{\log r} & =\varlimsup_{m \rightarrow \infty} L \frac{\sum_{s=m_{3}}^{m} s !}{m !} \frac{\log r_{m}}{\log r_{m}^{\prime}} \\
& =L \varlimsup_{m \rightarrow \infty} \frac{\log r_{m}}{\log r_{m}^{\prime}}
\end{aligned}
$$




$$
\begin{aligned}
& =L \varlimsup_{m \rightarrow \infty} \frac{\log r_{m}}{\log r_{m}+\left(\mu_{m} / \alpha_{m}\right) \log \left(r_{m+1} / r_{m}\right)} \\
& =L \varlimsup_{m \rightarrow \infty} \frac{1}{1+L / \alpha_{m}}=\frac{\alpha L}{\alpha+L}=\rho .
\end{aligned}
$$

Next, we set

and define

$$
K_{m}=r_{m+1} / r_{m}=3^{m \cdot m !}
$$

$$
K_{m}=\left(\log K_{m}^{\prime}\right)^{2 / \mu_{m}} .
$$

It is easy to see that $r_{m}^{\prime} / K_{m}>K_{m} r_{m}\left(m \geqq m_{4}\right)$. Here we estimate the size of the set

$$
F=\bigcup_{m=m_{4}}^{\infty}\left[K_{m} r_{m}, r_{m}^{\prime} / K_{m}\right]
$$

LEMMA 6. $\overline{\log \operatorname{dens}} F \geqq \rho / \alpha$.

Proof. Put $R=r_{m}^{\prime} / K_{m}\left(m \geqq m_{4}\right)$. Then

$$
\begin{aligned}
\int_{F \cap[1, R]} \frac{d t}{t} & =\sum_{s=m_{4}}^{m} \int_{K_{s} r_{s}}^{r_{s}^{\prime} / K_{s}} \frac{d t}{t} \\
& =\sum_{s=m_{4}}^{m}\left\{\frac{\mu_{s}}{\alpha_{s}} \log K_{s}^{\prime}-2 \log K_{s}\right\}>\frac{1-\varepsilon}{\alpha} \sum_{s=m_{5}}^{m} \mu_{s} \log K_{s}^{\prime} \quad\left(s \geqq m_{5}(\varepsilon)\right) \\
& =\frac{L(1-\varepsilon)}{\alpha} \log 3 \sum_{s=m_{5}}^{m} s !
\end{aligned}
$$

so that

Thus

$$
\begin{aligned}
\frac{1}{\log R} \int_{F \cap[1, R]} \frac{d t}{t} & >\frac{L(1-\varepsilon)}{\alpha} \log 3 \frac{\sum_{s=m_{5}}^{m} s !}{\log r_{m}^{\prime}-\log K_{m}} \\
& >\frac{L(1-\varepsilon)}{\alpha}-\frac{\sum_{s=m_{5}}^{m} s !}{m !+\left(L / \alpha_{m}\right) m !}=\frac{L(1-\varepsilon) \sum_{s=m_{5}}^{m} s !}{\left\{\alpha+L\left(\alpha / \alpha_{m}\right)\right\} m !}
\end{aligned}
$$

$$
\overline{\log \operatorname{dens}} F \geqq(1-\varepsilon) \rho / \alpha .
$$

Since $\varepsilon$ is an arbitrary positive number independent of $F$, we have

$$
\overline{\log \operatorname{dens}} F \geqq \rho / \alpha \text {. }
$$

Now, set $n(t)=0\left(t<r_{m_{3}}\right),=[\nu(t)]\left(t \geqq r_{m_{3}}\right)$, and define $f(z)$ as in $\S 3$. In this case, $f(z)$ satisfies

$$
\log f(z)=z \int_{r_{m_{3}}}^{\infty} \frac{n(t)}{t(t+z)} d t \quad(|\arg z|<\pi)
$$


LEMMA 7. $f(z)$ has order $\rho$ and lower order 0 .

Proof. As in the proof of Lemma 3, we can easily see that $f(z)$ has order $\rho$. We prove that the lower order of $f(z)$ is equal to 0 . Set $R=r_{m} / K_{m-1} \equiv R_{m}$, and write $\log M(R, f)$ as (3.4). Then

$$
\begin{aligned}
I_{3} & \leqq \frac{1}{1-\alpha_{1}}\left(\frac{1}{K_{m-1}}\right)^{1-\alpha_{1}} \nu\left(r_{m}\right) \quad\left(m \geqq m_{6}\right) \\
I_{2} & \sim\left(\log K_{m-1}^{\prime}\right) \nu\left(r_{m}\right) \quad(m \rightarrow \infty) \\
I_{1} & <\frac{\nu\left(r_{m}\right)}{\alpha_{m-1}}+\left(\log K_{m-2}^{\prime}\right) \sum_{s=m_{3}+1}^{m-1} \nu\left(r_{s}\right) \\
& =\nu\left(r_{m}\right)\left[\frac{1}{\alpha_{m-1}}+\left(\log K_{m-2}^{\prime}\right) \sum_{s=m_{3}+1}^{m-1}\left\{\frac{\nu\left(r_{s}\right)}{\nu\left(r_{m}\right)}\right\}\right] \\
& =\nu\left(r_{m}\right)\left[\frac{1}{\alpha_{m-1}}+\left(\log K_{m-2}^{\prime}\right) \sum_{s=m_{3}+1}^{m-1} e^{-L(\log 3)}{ }_{l=s}^{\left.\sum_{l}^{-1} l !\right]}\right] \\
& <\nu\left(r_{m}\right)\left[\frac{1}{\alpha_{m-1}}+\left(m-m_{3}-1\right) e^{-L(\log 3)(m-1) !}\left(\log K_{m-2}^{\prime}\right)\right] .
\end{aligned}
$$

Thus

$$
\begin{aligned}
& \log M(R, f)<\nu\left(r_{m}\right) O\left(\log K_{m-1}^{\prime}\right) \quad(m \rightarrow \infty), \\
& \frac{\log \log M(R, f)}{\log R}<\frac{\log \nu\left(r_{m}\right)+O\left(\log \log K_{m-1}^{\prime}\right)}{\log r_{m}-\log K_{m-1}} \\
& \leqq(1+o(1)) \frac{\log \nu\left(r_{m}\right)}{\log r_{m}}=(1+o(1)) \frac{L \sum_{s=m_{3}}^{m-1} s !}{m !} \longrightarrow 0 \\
& (m \rightarrow \infty) \text {. }
\end{aligned}
$$

Finally we modify the argument of the proof of Lemma 3 in [4] to obtain for $K_{m} r_{m} \leqq r \leqq r_{m}^{\prime} / K_{m}\left(m \geqq m_{3}\right)$

$$
\begin{aligned}
& \log M(r, f) \geqq \nu(r)\left\{\frac{\pi}{\sin \pi \alpha_{m}}+O\left(\frac{m}{\log K_{m}^{\prime}}\right)\right\}+O(\log r), \\
& \log m^{*}(r, f) \leqq \nu(r)\left\{\pi \cot \pi \alpha_{m}+O\left(\frac{m}{\log K_{m}^{\prime}}\right)\right\}+O(\log r)
\end{aligned}
$$

Thus

$$
\begin{aligned}
\frac{\log m *(r, f)}{\log M(r, f)} \leqq & \cos \pi \alpha_{m}+O\left(\frac{m}{\log K_{m}^{\prime}}\right)+O\left(-\frac{\log r}{2(r)}\right) \\
& =\cos \pi \alpha_{m}+O\left(\frac{1}{m !}\right) \\
& =\cos \pi \alpha_{m}+o\left(\alpha_{m}-\alpha\right)<\cos \pi \alpha \quad\left(r \in F \cap\left[r_{m_{7}}, \infty\right)\right) .
\end{aligned}
$$


From this and Lemma 6 we deduce that

$$
\underline{\log \text { dens }} E \leqq 1-\rho / \alpha .
$$

On the other hand, from Lemma 7 and Theorem A or Theorem B it follows that

$$
1-\rho / \alpha \leqq \underline{\log \operatorname{dens}} E<\overline{\log \operatorname{dens}} E=1 .
$$

Hence

$$
1-\rho / \alpha=\underline{\log \operatorname{dens}} E<\overline{\log \operatorname{dens}} E=1 .
$$

This completes the proof.

\section{REFERENCES}

[1] BARRY, P. D., On a theorem of Besicovitch, Quart. J. Math. Oxford (2), 14 (1963), 293-302.

[2] BARRY, P. D., On a theorem of Kjellberg, ibid. (2), 15 (1964), 179-191.

[3] Hayman, W. K., Meromorphic functions, Oxford, Clarendon Press, (1964).

[4] Hayman, W.K., Some examples related to the $\cos \pi \rho$ theorem, Mathematical essays dedicated to A. J. Macintyre, Ohio Univ. Press (1970), 149-170.

[5] KJellberG, B., On the minimum modulus of entire functions of lower order less than one, Math. Scand. 8 (1960), 189-197.

Department of Mathematics

Daido Institute of Technology

DAIDO-Cho, MinAMI-KU, NAGOYA, JAPAN 